\title{
DYNAMICS OF MULTIPLE-DEGREE-OF-FREEDOM OSCILLATORS WITH COLLIDING COMPONENTS
}

\author{
S. Natsiavas \\ Department of Mechanical and Aerospace Engineering, Arizona State University, Tempe, \\ Arizona 85287 , U.S.A.
}

\begin{abstract}
An analysis is presented for determining exact steady state response for a class of strongly non-linear multiple-degree-of-freedom oscillators. These oscillators consist of a linear component, with an arbitrary number of degrees of freedom and configuration, incorporating a component with a geometric non-linearity. Their behavior is analyzed by generalizing previous work on single- and two-degree-of-freedom piecewise linear systems under harmonic excitation. First, a methodology is developed for locating harmonic and subharmonic response characterized by one abrupt change of the parameters of the nonlinear element per response cycle. Then, a stability analysis is presented, which is appropriate for general piecewise linear systems. The present analysis is applicable to a variety of engineering areas. Here, its validity and effectiveness is demonstrated by applying it to two classes of problems with large practical significance and rich dynamics. Performance of vibration absorbers with elastic stops is investigated first, while in the second category of examples the oscillators exhibit $2: 1$ and $3: 1$ internal resonance. Regular and irregular motions are encountered and analyzed. The response characteristics are compared with those of systems with continuous non-linearities.
\end{abstract}

\section{INTRODUCTION}

In a large number of diverse engineering fields, design or operational conditions lead to collisions between the components of a system. Examples of this type include gears, bearings, vibration isolation elements, piping systems, offshore structures, pounding of adjacent structures during earthquakes, hammer impact tools and impact dampers. The large interest in analyzing and understanding the performance of such systems is reflected by the vast and ever increasing amount of research effort devoted in this area, a small sample of which is reported in references [1-14]. The characteristics of these systems are inherently non-linear. Therefore, application of standard analytical tools based on linearizations will fail to capture essential ingredients of the response. On the other hand, the presence of the non-linearities complicates the analysis considerably. As a result, the majority of the previous work idealizes these systems as single-degree-of-freedom oscillators. Exceptions are references $[1-5,8,11]$, most of which analyze two-degree-of-freedom systems. Finally, references [2] and [5] study the response of multi-degree systems with impact dampers, by assuming damping leading to classical normal modes and without examining stability.

The present analysis extends the previous work on piecewise linear oscillators by developing a systematic and computationally efficient way of determining exact periodic motions for a general multiple degree of freedom system, with a component that possesses piecewise linear damping and restoring forces. Both the existence and stability properties of a harmonic and subharmonic response characterized by a single "collision" (when damping and/or stiffness parameters change) within a response period are analyzed. This is achieved 
by proceeding along the lines of the analysis for similar single-degree-of-freedom oscillators $[13,14]$. This type of analysis exploits the linearity of the equations of motion within each time interval where the system possesses constant characteristics. As expected [10], this extension is not a trivial task. A key feature that permits this generalization is the derivation of a suitable expression for the form of the solution of the linear problem.

The basic steps of the present analysis are briefly outlined in the following three sections. First, a suitable solution form is obtained for a general discrete linear system under harmonic excitation. This solution form then presents the basis for determining exact periodic motions of the non-linear system, as well as their stability. The analysis developed can be applied in a number of different areas. Here, the validity and effectiveness of the method is tested by applying it to two classes of important engineering problems. In this way, useful information is also obtained for the dynamics of the examples considered, which are vibration absorbers with elastic stops and other oscillators under internal resonance. The results of the numerical study reveal that some parameter combinations lead to a periodic or quasi-periodic response, while others lead to chaotically modulated motions. Comparison with the response of oscillators with continuous non-linearities is also made.

\section{MECHANICAL MODEL AND SOLUTION FORM}

The system examined is modeled as a general linear multiple-degree-of-freedom oscillator, including a component carrying elastic stops with damping and restoring forces, expressed by

$$
F(\dot{x}, x)= \begin{cases}c \dot{x}+k x, & x \leqslant x_{s}, \\ c^{\prime} \dot{x}+k^{\prime} x+\left(k-k^{\prime}\right) x_{s}, & x>x_{s} .\end{cases}
$$

In equation (1), $x$ is the relative displacement of the oscillator carrying the stops with respect to the component of the system these stops collide with. An example of such a system with $c=c_{N}, c^{\prime}=c_{N}+c_{s}, k=k_{N}$ and $k^{\prime}=k_{N}+k_{s}$ is presented in Figure 1. In general, the position of the stops within the overall system can be arbitrary.

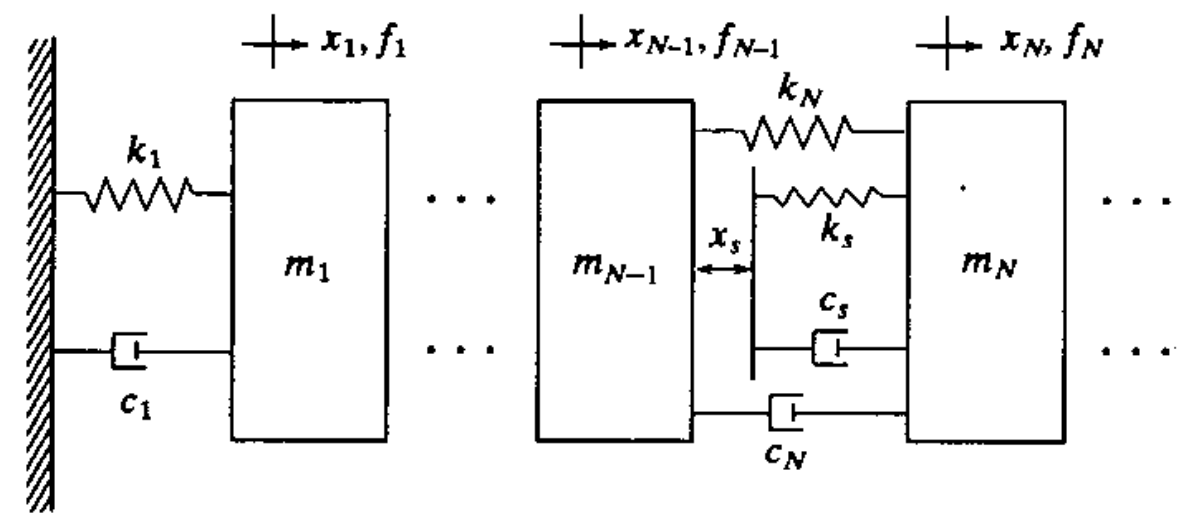

Figure 1. A mechanical model of the system.

The components of the system are subjected to forcing: $f_{i}(t)=F_{i} \cos \left(\omega t+\phi_{i}+\phi\right)$, where $\phi$ is an unknown phase angle introduced for the purpose of facilitating the search of periodic solutions. Since the function $F(\dot{x}, x)$ is piecewise linear, the exact form of the analytical solution of the system can be obtained for $x \geqslant x_{s}$ and $x<x_{s}$. Before doing this, the displacements are normalized by a characteristic length, $x_{c}$, i.e., $y_{i}=x_{i} / x_{c}$. This length can be the gap length $x_{s}$, when $x_{s}$ is non-zero. In addition, a normalized time is chosen according to $\tau=\omega t$. Then, the governing equations in the time intervals where the stiffness 
and damping remain constant can be cast in the linear form

$$
M \ddot{\mathbf{y}}+C \dot{\mathbf{y}}+K \mathbf{y}=\mathbf{f}(\tau)+\mathbf{k},
$$

where $M, C$ and $K$ are the inertia, damping and stiffness matrices, respectively, while $\mathbf{k}$ is a vector including the constant terms of equation (1). Therefore, the general solution in the interval considered is

$$
\mathbf{y}=\mathbf{y}_{h}+\mathbf{y}_{\rho}
$$

The homogeneous part, $\mathbf{y}_{h}$, is expressed in terms of the eigenvalues $s_{n}=\rho_{n}+\mathrm{i} \sigma_{n}$ and the corresponding eigenvectors $\hat{\mathbf{y}}_{n}=\boldsymbol{a}_{n}+\mathrm{i} \boldsymbol{\beta}_{n}$, determined by

$$
\left(s_{n}^{2} M+s_{n} C+K\right) \hat{\mathbf{y}}_{n}=\mathbf{0}, \quad n=1, \ldots, N,
$$

where $N$ is the total number of degrees of freedom. Note that the solution of the above eigenvalue problem is equivalent to that obtained by converting equation (2) to the classical first order $2 N$-space form. For oscillatory systems, the $s_{n}$ 's and $\hat{\mathbf{y}}_{n}$ 's appear in complex conjugate pairs. Making use of this, $\mathbf{y}_{h}$ is expressed in terms of real quantities as

$$
\mathbf{y}_{h}(\tau)=\sum_{n=1}^{N} A_{n}(\tau) \mathbf{c}_{n}
$$

where the $N \times 2$ matrix $A_{n}$ is defined by

$$
A_{n}(\tau)=\mathrm{e}^{\rho_{n} \tau}\left[B_{n} \cos \left(\sigma_{n} \tau\right)+C_{n} \sin \left(\sigma_{n} \tau\right)\right],
$$

while $B_{n}=\left[\begin{array}{ll}\boldsymbol{\alpha}_{n} & \boldsymbol{\beta}_{n}\end{array}\right], C_{n}=\left[\begin{array}{ll}-\boldsymbol{\beta}_{n} & \boldsymbol{\alpha}_{n}\end{array}\right]$ and the constants $\boldsymbol{c}_{n}$ are determined by satisfying initial conditions.

Next, the forcing vector $\mathbf{f}(\tau)$ is split in the form: $\mathbf{f}_{c} \cos (\tau+\phi)+\mathbf{f}_{s} \sin (\tau+\phi)$, and the particular solution of equation (2) is determined in the form

$$
\mathbf{y}_{p}(\tau)=\mathbf{y}_{c} \cos (\tau+\phi)+\mathbf{y}_{s} \sin (\tau+\phi)+\mathbf{p}
$$

from

$$
\left[\begin{array}{cc}
K-M & C \\
C & M-K
\end{array}\right]\left(\begin{array}{l}
\mathbf{y}_{c} \\
\mathbf{y}_{s}
\end{array}\right)=\left(\begin{array}{c}
\mathbf{f}_{c} \\
-\mathbf{f}_{s}
\end{array}\right), \quad K \mathbf{p}=\mathbf{k}
$$

Then, the particular equation is rewritten in the form

$$
\mathbf{y}_{p}(\tau)=\mathbf{g}(\tau) \cos \phi+\mathbf{h}(\tau) \sin \phi+\mathbf{p}
$$

Introducing the vector

$$
\mathbf{z}=\left[\begin{array}{ll}
\dot{\mathbf{y}} & \mathbf{y}
\end{array}\right]^{\mathbf{T}},
$$

and combining all the above, the solution is finally expressed in the form

$$
\mathbf{z}(\tau)=\Phi(\tau) \gamma+\mu(\tau) \cos \phi+\mathrm{v}(\tau) \sin \phi+\xi,
$$

where

$$
\Phi=\left(\begin{array}{lll}
\dot{A}_{1} & \cdots & \dot{A}_{N} \\
A_{1} & \cdots & A_{N}
\end{array}\right), \quad \mu=\left(\begin{array}{l}
\dot{\mathbf{g}} \\
\mathbf{g}
\end{array}\right), \quad \mathrm{v}=\left(\begin{array}{l}
\dot{\mathbf{h}} \\
\mathbf{h}
\end{array}\right), \quad \xi=\left(\begin{array}{l}
\mathbf{0} \\
\mathbf{p}
\end{array}\right),
$$

and $\gamma=\left[\mathbf{c}_{1} \cdots \mathbf{c}_{N}\right]^{\mathrm{T}}$. 


\section{PERIODIC STEADY STATE VIBRATIONS}

Based on the solution form (4), a methodology is presented in this section, which leads to exact, $n$-periodic solutions of the system examined in a systematic and efficient way. These solutions are characterized by a single change of the damping and stiffness parameters within a response cycle. Since the system is non-linear, other vibrational modes are also possible. However, the motions considered are expected to be the most dominant. Then, the form of the solution sought is dictated by the form of $u=y_{N}$, which is depicted in Figure 2: namely, the solution is $\mathbf{z}_{1}\left(\tau_{1}\right)$ in $0 \leqslant \tau_{1}=\tau \leqslant \tau_{1}^{*}$ and $\mathbf{z}_{2}\left(\tau_{2}\right)$ in $0 \leqslant \tau_{2}=\tau-\tau_{1}^{*} \leqslant \tau_{2}^{*}$, where $\tau_{1}^{*}$ is the first contact time and $\tau_{2}^{*}=2 \pi n-\tau_{1}^{*}$.

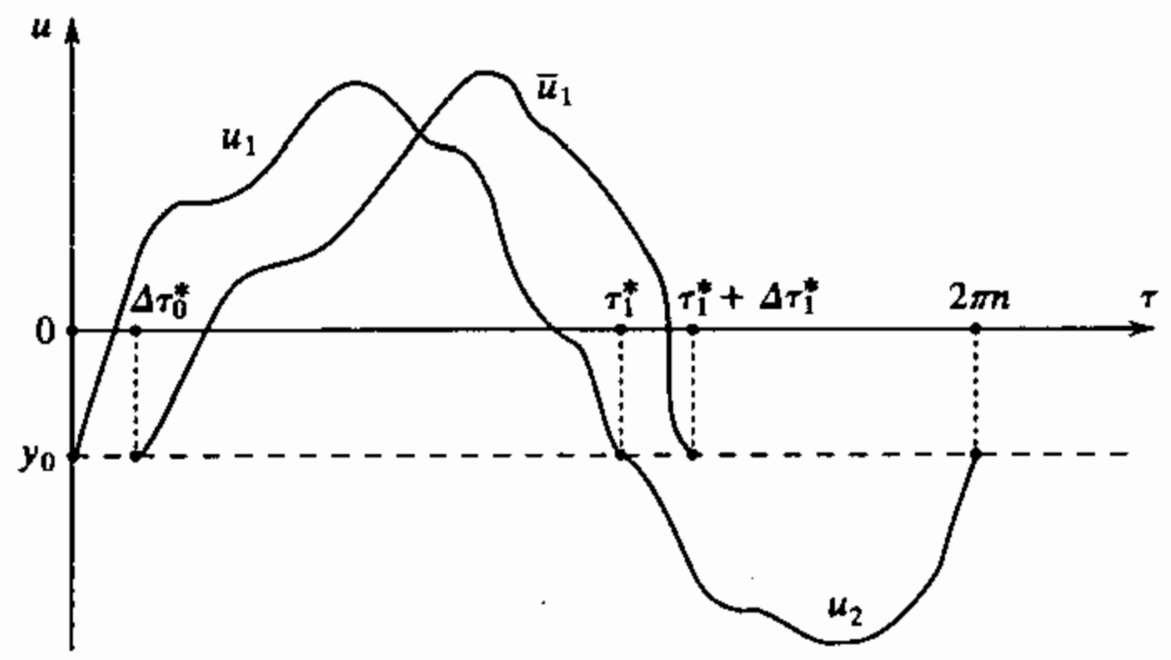

Figure 2. The solution form of $u=y_{N}$.

The determination of the desired solutions is based on the fact that they satisfy the following set of periodicity and matching conditions:

$$
\begin{gathered}
\mathbf{z}_{1}(0)=\mathbf{z}_{2}\left(\tau_{2}^{*}\right), \quad \mathbf{z}_{1}\left(\tau_{1}^{*}\right)=\mathbf{z}_{2}(0), \\
u_{1}(0)=u_{2}(0)=y_{0} \equiv x_{s} / x_{c} .
\end{gathered}
$$

The above represent a set of $4 N+2$ transcendental equations for the $4 N+2$ unknowns of the problem (i.e., $\gamma_{1}, \gamma_{2}, \tau_{1}^{*}$ and $\phi$ ). However, generalizing a solution procedure developed for similar but single-degree-of-freedom oscillators [13, 14], the task of solving these equations can eventually be reduced to the solution of a single transcendental equation, as explained next.

First, application of equation (5) leads to

$$
\left[\begin{array}{cc}
\Phi_{1}(0) & -\Phi_{2}\left(\tau_{2}^{*}\right) \\
\Phi_{1}\left(\tau_{1}^{*}\right) & -\Phi_{2}(0)
\end{array}\right] \mathbf{w}=\left(\begin{array}{l}
\mu_{2}\left(\tau_{2}^{*}\right)-\mu_{1}(0) \\
\mu_{2}(0)-\mu_{1}\left(\tau_{1}^{*}\right)
\end{array}\right) \cos \phi+\left(\begin{array}{l}
v_{2}\left(\tau_{2}^{*}\right)-v_{1}(0) \\
\boldsymbol{v}_{2}(0)-v_{1}\left(\tau_{1}^{*}\right)
\end{array}\right) \sin \phi+\left(\begin{array}{l}
\xi_{2} \\
\xi_{2}
\end{array}\right)
$$

where $w=\left[\begin{array}{ll}\gamma_{1} & \gamma_{2}\end{array}\right]^{\top}$. Note that for the solution form of Figure $2, \xi_{1} \equiv \mathbf{0}$. Next, for a given value of $\tau_{1}^{*}$, solution of equation (7) provides the vector $w$ in the form

$$
\mathbf{w}=\mathbf{w}_{c} \cos \phi+\mathbf{w}_{s} \sin \phi+\mathbf{w}_{0} .
$$

Then, application of equation (6) leads to two algebraic equations of the form

$$
a_{s} \sin \phi+a_{c} \cos \phi=a_{0}, \quad b_{s} \sin \phi+b_{c} \cos \phi=b_{0} .
$$


Solving equations (9) and (10) for $\sin \phi$ and $\cos \phi$ and applying a trigonometric identity, the correct value of $\tau_{1}^{*}$ will give rise to

$$
G\left(\tau_{1}^{*}\right) \equiv\left(a_{0} b_{c}-a_{c} b_{0}\right)^{2}+\left(a_{s} b_{0}-a_{0} b_{s}\right)^{2}-\left(a_{s} b_{c}-a_{c} b_{s}\right)^{2}=0 .
$$

The strategy leading to a complete numerical solution of the problem is based on the observation that all the matrices and vectors of equation (7), as well as the $a$ 's and $b$ 's in equations (9) and (10), are functions of the system parameters and $\tau_{1}^{*}$ only. Moreover, $0<\tau_{1}^{*}<2 \pi n$. Then, the solution procedure starts by assigning values to $\tau_{1}^{*}$ within the range from 0 to $2 \pi n$. This permits the determination of $w$ from equation (7) and the evaluation of the corresponding value of $G$ from equation (11), independently of the value of $\phi$. In doing so, the bisection method is applied in locating a possible solution for $\tau_{1}^{*}$, when a sign change of $G$ is detected between subsequent trial values of $\tau_{1}^{*}$. Determination of $\tau_{1}^{*}$ allows the calculation of $\phi$ from equations (9) and (10), the evaluation of the $w$ from equation (8) and therefore the solution histories of $z_{1}$ and $z_{2}$ from equation (4).

The above methodology can be modified and applied to other important cases, including systems with symmetric characteristics.

\section{STABILITY OF PERIODIC RESPONSE}

The information about the stability properties of a steady state motion is important because only stable solutions are realizable in practice. Moreover, the way in which a solution loses stability presents useful information in searching for other possible motions that may coexist. For piecewise linear systems, the classical methodologies are not applicable in performing the stability analysis of located periodic solutions. Here, a method employed before for other similar oscillators with one and two degrees of freedom will be generalized and applied.

Following reference [12], the essential information about the stability of a solution of a piecewise linear oscillator is obtained by just analyzing the propagation of arbitrary perturbations of the starting conditions within a single interval of two successive contact times. This actually implies that the steps of the analysis that follows are general and can be applied to solutions of piecewise linear systems of arbitrary form.

The stability procedure starts by letting $\Delta \tau_{1}=\Delta \tau_{1}^{*}-\Delta \tau_{0}^{*}$, where $\Delta$ denotes a small perturbation of the variable it is applied on. Next, consider an exact periodic solution $\mathbf{z}_{1}$ and a perturbed solution $\overline{\mathbf{z}}_{1}$, obtained by perturbing the initial conditions of $\mathbf{z}_{1}$ slightly (see Figure 2). Then,

$$
\overline{\mathbf{z}}_{1}\left(\tau_{1}^{*}+\Delta \tau_{1}\right)=\mathbf{z}_{1}\left(\tau_{1}^{*}\right)+\Delta \mathbf{z}_{1},
$$

where the vector $\Delta \mathbf{z}_{1}$ represents the deviation of $\overline{\mathbf{z}}_{1}$ from $\mathbf{z}_{1}$ at time $\tau_{1}^{*}$. Note that the corresponding variation of $u_{1}$ is zero and, therefore, its position in $\Delta z_{1}$ is filled by the time variation $\Delta \tau_{1}^{*}$ instead. Application of equation (12) yields

$$
\Phi_{1}\left(\tau_{1}^{*}+\Delta \tau_{1}\right) \gamma_{1}\left(\mathbf{z}_{0}+\Delta \mathbf{z}_{0}\right)+\mu\left(\tau_{1}^{*}+\Delta \tau_{1}^{*}\right) \cos \phi+\mathbf{v}\left(\tau_{1}^{*}+\Delta \tau_{1}^{*}\right) \sin \phi=\mathbf{z}_{1}\left(\tau_{1}^{*}\right)+\Delta \mathbf{z}_{1},
$$

where the vector $\Delta \mathbf{z}_{0}$ involves the deviation from the correct initial conditions, $\mathbf{z}_{0}=\mathbf{z}_{1}(0)$, leading to $z_{1}$. These initial conditions are determined by letting $\tau_{1}=0$ in equation (4), once a periodic motion has been located by the analysis of the previous section. Next, expanding the left side of the last equation and keeping up to first order terms leads to

$$
\Phi_{1}\left(\tau_{1}^{*}\right) \Gamma_{1} \Delta \mathrm{z}_{0}+\dot{\Phi}_{1}\left(\tau_{1}^{*}\right) \gamma_{1} \Delta \tau_{1}+\dot{\mathbf{q}}_{1}\left(\tau_{1}^{*}\right) \Delta \tau_{1}^{*}=\Delta \mathrm{z}_{1}
$$


where $\boldsymbol{q}_{1}\left(\tau_{1}\right)=\boldsymbol{\mu}_{1}\left(\tau_{1}\right) \cos \phi+\boldsymbol{v}_{1}\left(\tau_{1}\right) \sin \phi$, and the matrix $\Gamma_{1}$ is formed by adding the vector $\Phi_{1}^{-1}(0) \dot{q}_{1}(0)$ in the last column of the matrix $\Phi_{1}^{-1}(0)$. The zeroth order terms drop out because they satisfy equation (5). Then, the last equation can be put in the form

$$
\Delta \mathbf{z}_{1}=Q_{1} \Delta \mathbf{z}_{0},
$$

with $Q_{1}=R_{11}^{-1} R_{10}$. The matrix $R_{11}$ is formed by adding the vector $-\left[\dot{\Phi}_{1}\left(\tau_{1}^{*}\right) \gamma_{1}+\dot{\mathbf{q}}_{1}\left(\tau_{1}^{*}\right)\right]$ in the last column of the identity matrix of order $2 N \times 2 N$, while $R_{10}$ is obtained by subtracting the vector $\dot{\Phi}_{1}\left(\tau_{1}^{*}\right) \gamma_{1}$ from the last column of the matrix $\Phi_{1}\left(\tau_{1}^{*}\right) \Gamma_{1}$.

Proceeding in a similar fashion in the interval $0<\tau_{2}<\tau_{2}^{*}$ yields

$$
\Delta \mathbf{z}_{2}=Q_{2} \Delta \mathbf{z}_{1},
$$

where, again, the matrix $Q_{2}$ is found in terms of the system parameters only. Then, the relation between the perturbations at the beginning and at the end of the first response period is expressed by

$$
\Delta \mathbf{z}_{2}=\Pi \Delta \mathbf{z}_{0}
$$

where $\Pi=Q_{2} Q_{1}$. Finally, after $m$ response cycles, the perturbations from the correct initial conditions will be related to first order with the original perturbations by

$$
\Delta \mathrm{z}_{2 m}=\Pi^{m} \Delta \mathrm{z}_{0} .
$$

Therefore, if $\lambda$ is the eigenvalue of $\Pi$ with the largest modulus, the effect of the original error will vanish with time, provided that $|\lambda|<1$. In such cases, the periodic solution examined is asymptotically stable. When $|\lambda|>1$, the solution is unstable. Finally, when $|\lambda|=1$, bifurcations occur, resulting in qualitative changes of the system dynamics [15]. According to the results presented in the next section, three types of bifurcations were encountered in this study. The first type is a saddle-node bifurcation: it occurs with $\lambda=1$ and results in the classical jump phenomena. The second type happens with $\lambda=-1$ and is associated with period doubling of the response. The third type is the so-called Hopf bifurcation, it occurs when a pair of complex eigenvalues has modulus one and results in an amplitude modulated response of the system. Note that if the damping coefficients are non-negative, the Hopf bifurcation is excluded for single degree of freedom oscillators [7, 12], but it occurs for systems with $N \geqslant 2$.

\section{APPLICATIONS}

This section verifies the analysis developed by presenting results for systems with two degrees of freedom (the system shown in Figure 1 with $N=2$ ). The mass $m_{1}$ is subjected to a forcing $F_{1} \cos \tau$, while $F_{2}=0$. For convenience, introduce the additional dimensionless parameters

$$
\bar{\omega}_{i}=\sqrt{k_{i} / m_{i}}, \quad \omega_{i}=\bar{\omega}_{i} / \omega, \quad \zeta_{i}=c_{i} /\left(2 \sqrt{k_{i} m_{i}}\right), \quad \eta_{i}=\omega_{i}^{2}, \quad \delta_{i}=2 \zeta_{i} \omega_{i} \quad(i=1,2),
$$

with similar relationships holding for the primed variables (see equation (1) for their definition) and

$$
\beta=F_{1} /\left(k_{1} x_{c}\right), \quad \bar{\omega}=\omega / \bar{\omega}_{1}, \quad \rho=\omega_{2} / \omega_{1}, \quad \rho^{\prime}=\omega_{2}^{\prime} / \omega_{1}, \quad \mu=m_{2} / m_{1} .
$$


Then, the inertia, damping and stiffness matrices and the forcing vectors are expressed by

$$
\begin{gathered}
M=\left[\begin{array}{cc}
1+\mu & \mu \\
\mu & \mu
\end{array}\right], \quad C=\left[\begin{array}{cc}
\delta_{1} & 0 \\
0 & \mu \hat{\delta}_{2}
\end{array}\right], \quad K=\left[\begin{array}{cc}
\eta_{1} & 0 \\
0 & \mu \hat{\eta}_{2}
\end{array}\right], \\
\mathbf{f}=\left(\begin{array}{l}
\beta \\
0
\end{array}\right) \eta_{1} \cos \tau, \quad \mathbf{k}=\left(\begin{array}{c}
0 \\
\mu \hat{k}_{2}
\end{array}\right),
\end{gathered}
$$

where

$$
\left(\hat{\delta}_{2}, \hat{\eta}_{2}, \hat{k}_{2}\right)= \begin{cases}\left(\delta_{2}, \eta_{2}, 0\right), & y_{2} \geqslant y_{0} \\ \left(\delta_{2}^{\prime}, \eta_{2}^{\prime},\left(\eta_{2}^{\prime}-\eta_{2}\right) y_{0}\right), & y_{2} \leqslant y_{0}\end{cases}
$$

The numerical results obtained for this system are presented in the following two subsections. In all cases considered, $y_{0}=-1$. For other systems, the only difference in the analysis will be in the form of $M, C, K, \mathbf{f}$ and $\mathbf{k}$.

\subsection{VIBRATION ABSORBERS WITH ELASTIC STOPS}

The idea of using stops in vibration absorption was presented in earlier studies $[16,1]$. The main interest there was to limit excessive amplitudes of vibration of the auxiliary mass of the absorber. Here, the analysis developed is applied in order to show some further enhancements and some limitations in the performance of the absorber with stops.

First, in Figure 3 is presented the response diagram of the main mass for three systems with parameters $\mu=0 \cdot 1, \beta=0 \cdot 1, \rho=1, \zeta_{1}=0.01$ and $\zeta_{2}=0 \cdot 02$. The influence of the stops is examined by choosing the values of $\rho^{\prime}$ to be $1,0.707$ and 1.414 , while the values of $\zeta_{2}^{\prime}$ are chosen as $0.02,0.0141$ and 0.0283 , respectively. These parameters represent a linear oscillator and two non-linear oscillators with stops. The stops provide the auxiliary mass with damping and stiffness coefficients equal to half and double their corresponding original (before stops come into action) values, respectively. Physically, the first system is

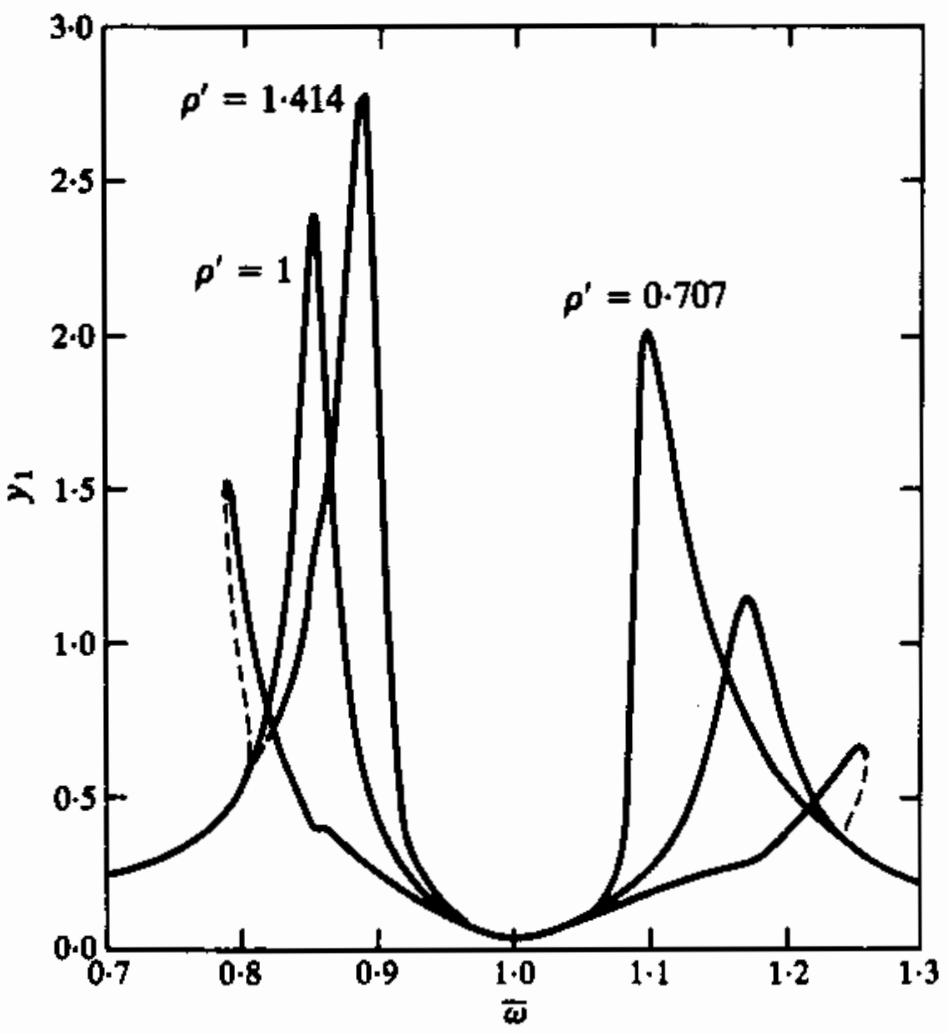

Figure 3. The response diagram of the main mass: $\rho^{\prime}=1,0.707$ and 1.414 . 
originally in pretension and loses partial contact at $y_{0}$ and, as a consequence, presents softening characteristics, while the second non-linear oscilla tor possesses hardening characteristics. The effectiveness of the stops is obvious, when comparing the response diagram of the linear absorber (thin line) with the softening/hardening absorber in the lower/ higher forcing frequency ranges, respectively. These benefits were expected from previous work on absorbers with continuous non-linearities (e.g., [17-19]). In fact, the present setup provides a practically feasible way of designing non-linear absorbers. However, in analogy to the non-linear absorber with continuous characteristics, the non-linearities also introduce some potential dangers, as explained next.

In Figure 3, the unstable branch of the softening oscillator is generated with $\lambda=1$ bifurcations, associated with jump phenomena of the response at $\bar{\omega}=0.789$ and 0.806 , and coexists with two other stable periodic solutions. Similar bifurcations are captured for the hardening system at $\bar{\omega}=1.244$ and 1.259 . In addition, the latter system exhibits period doubling $(\lambda=-1)$ bifurcations (at 0.805 and 0.816 ) as well as Hopf bifurcations (at 1.034 and 1.061).

Response diagrams obtained by raising the non-linearity level are presented in Figure 4. This is done by increasing $\rho^{\prime}$ to 1.732 and 4 , which corresponds to stops with twice and 15 times the original stiffness of the absorber (approaching conditions of impact dampers), respectively, while maintaining $\zeta_{2}^{\prime}=0.02$ and the values of the other parameters as before. All the bifurcations of the lower resonance branches occur with $\lambda=-1$, while $\lambda=1$ gives rise to the unstable motions of the higher resonance branches. Branch $C$ is common to both diagrams. From the last plot, the most important observation is that increasing the non-linearity level causes a significant expansion of the zone of the unstable solutions originated by Hopf bifurcations near the original resonance $\ddot{\omega}=1$. This is in accordance with results for absorbers with continuous non-linearities $[18,19]$ and gives rise to rich but dangerous dynamics within that frequency range. For example, in Figure 5 are shown samples of the Poincare sections of $y_{1} v s$. $\dot{y}_{2}$, for $\rho^{\prime}=4$. These plots were obtained by direct integration of the equations of motion at several values of $\bar{\omega}$ and by sampling the longtime response once every forcing cycle.

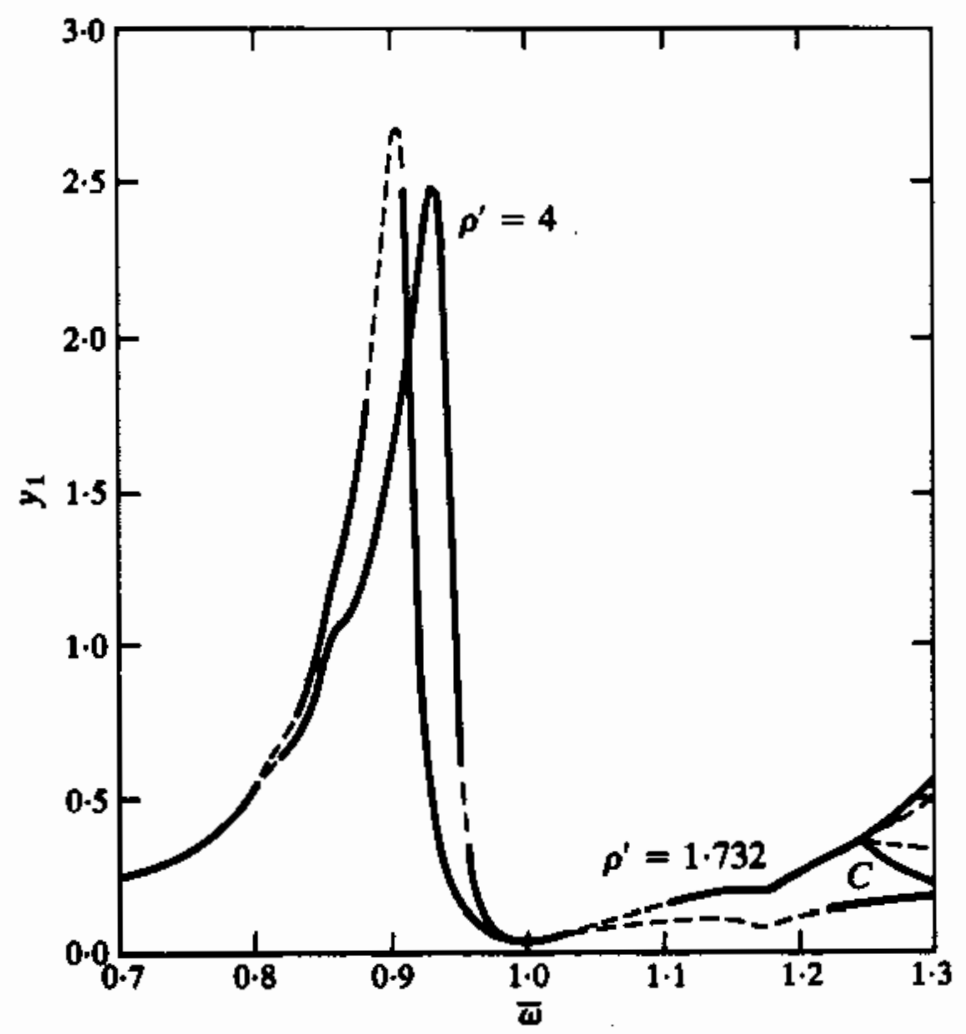

Figure 4. The response diagram of the main mass: $\rho^{\prime}=1.732$ and 4. 


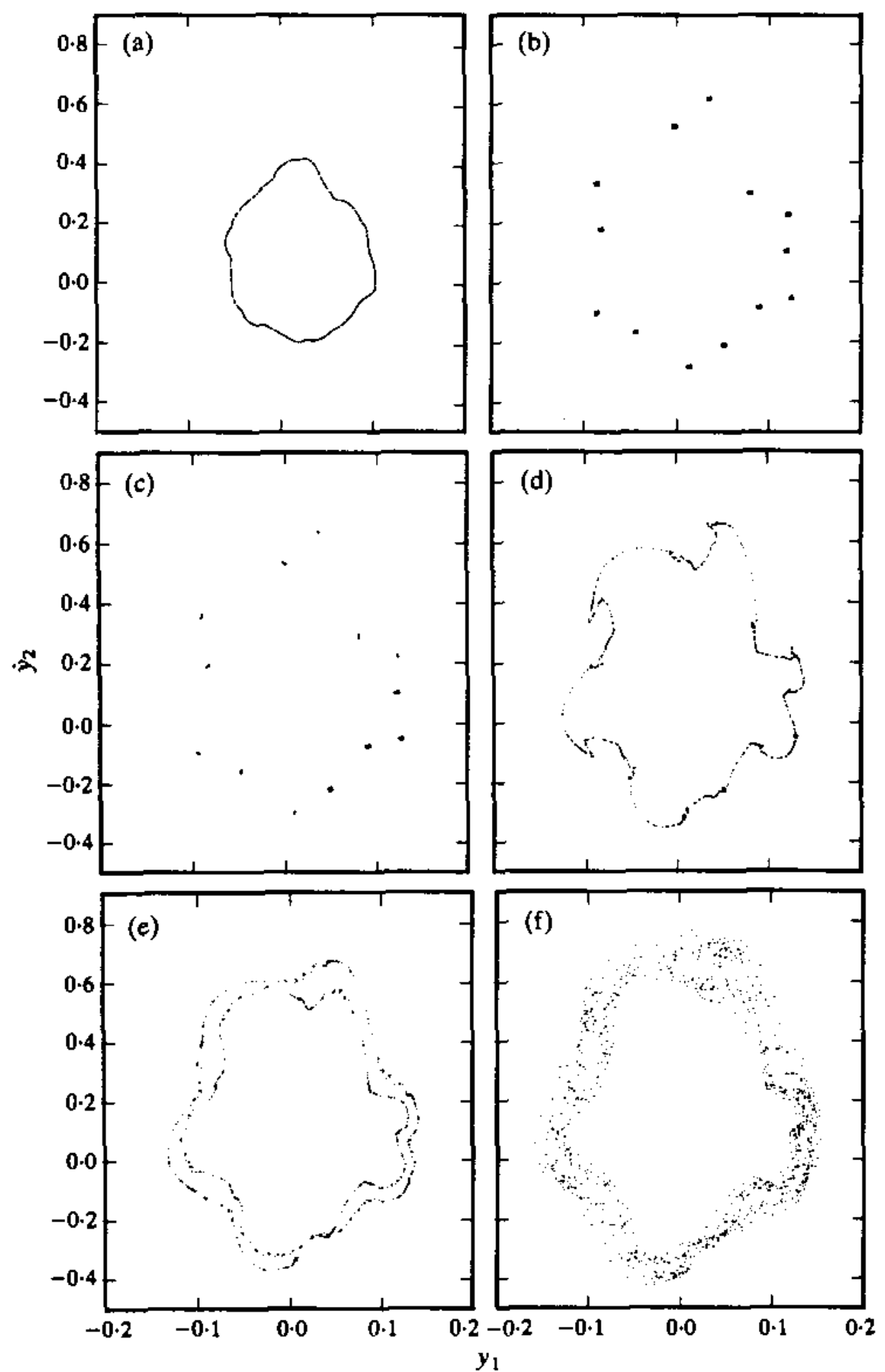

Figure 5. Poincaré sections: (a) $\bar{\omega}=1.0180 ;$ (b) $\bar{\omega}=1.0220$; (c) $\bar{\omega}=1.0225$; (d) $\bar{\omega}=1.0230 ;$ (e) $\bar{\omega}=1.0233$; (f) $\bar{\omega} 1 \cdot 0250$.

As expected, a quasi-periodic response, represented by the invariant circle of Figure 5(a), appears at $\bar{\omega}=1 \cdot 018$, just after the Hopf bifurcation value of 1.017 . This response becomes phase locked occasionally, as shown in Figure $5(\mathrm{~b})$ for the $n=13$ solution obtained at 1.022. As the value of $\bar{\omega}$ moves further away from the Hopf bifurcation value, the invariant circle expands, splits to $n=13$ attractors (Figure 5(c)) and distorts (Figure $5(d)$ ), according to a scenario explained in reference [20]. Then, following a single torus doubling (Figure 5(e)), the system settles into chaotic response (Figure 5(f)) without any further observable torus doubling (see references [21] and [22] for similar results and explanations).

To examine the effect of these motions on the performance of the absorber, in Figure 6(a) is shown the steady state response history of $y_{1}$ at $\bar{\omega}=1.018$, during 95 forcing 

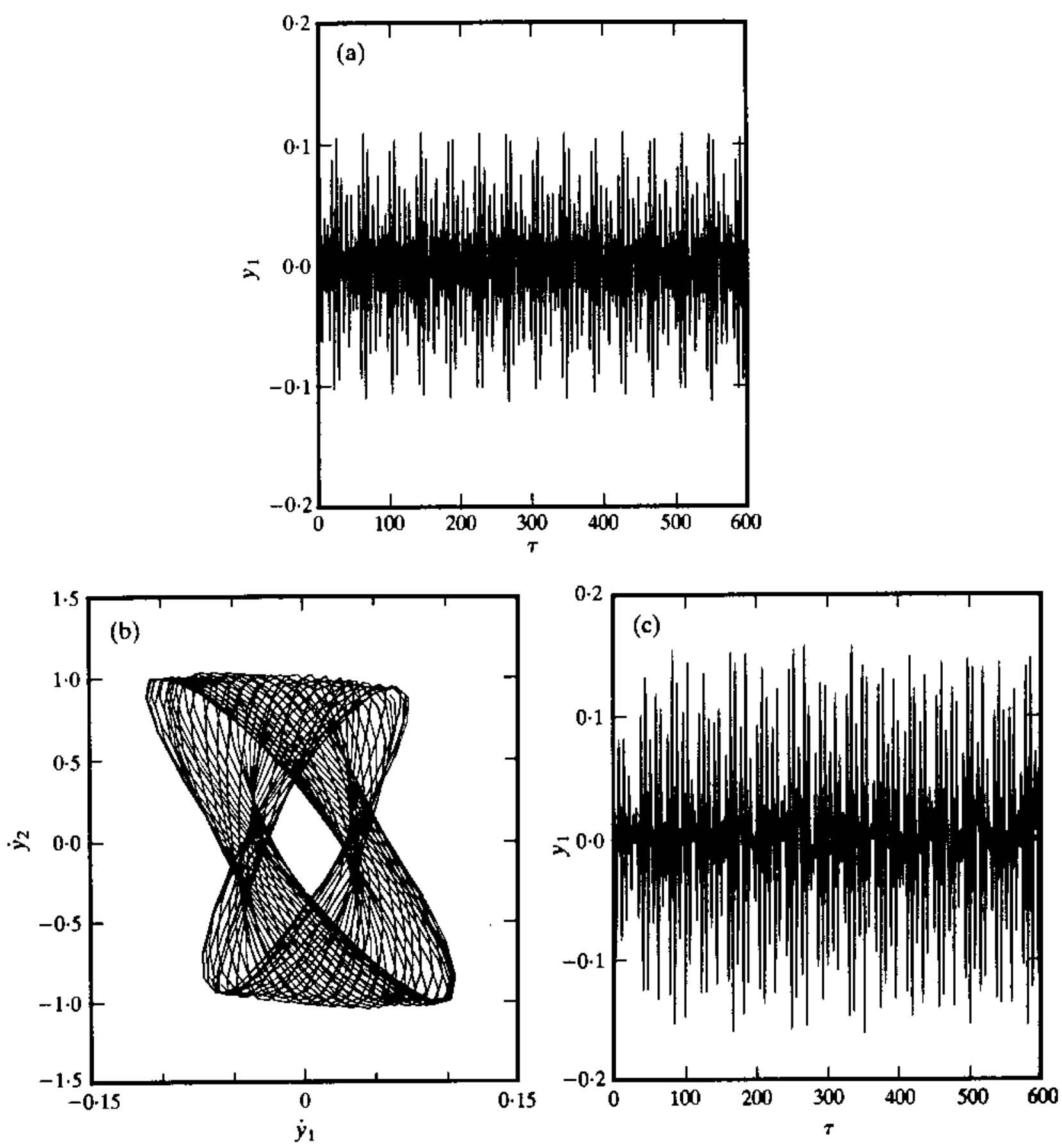

Figure 6. (a) The response history at $\bar{\omega}=1.018$; (b) projection onto the $\left(\dot{y}_{1}, \dot{y}_{2}\right)$ phase plane $(\bar{\omega}=1.018)$; (c) response history at $\bar{\omega}=1 \cdot 025$.

cycles. Clearly, the response involves beats, which is in agreement with previously reported experimental results [2,3]. In Figure 6(b) it is shown that this quasi-periodic motion rides on a torus in phase space co-ordinates, by plotting part of the trajectory in the phase plane of $\dot{y}_{1} v s . \dot{y}_{2}$, at the same value of $\bar{\omega}$. Finally, in Figure 6(c) are shown 95 forcing cycles of the long-time response history of $y_{1}$ at $\bar{\omega}=1.025$. This motion corresponds to that of Figure 5(f) and is chaotically modulated. The amplitude of the corresponding unstable periodic solutions at $\bar{\omega}=1.018$ and 1.025 is only 0.052 and 0.058 , respectively. Comparison shows that caution should be exercised in choosing the absorber parameters properly. The present analysis can then be used as a guide in adjusting the parameters and eliminating these unstable responses.

\subsection{OSCILLATORS WITH INTERNAL RESONANCE}

The systems examined above have "equivalent" linearized counterparts with close natural frequencies. In this section, it is shown that some of the phenomena observed for the 
non-linear absorber can also occur in other oscillatory systems, when their parameters are chosen in ways that lead to internal resonance conditions [23-26]. A number of some new and interesting features also appear and are analyzed.

First, in Figure 7 is presented the response diagram for a system with parameters $\mu=$ $\beta=0.5, \rho=0.9, \rho^{\prime}=0.65, \zeta_{1}=0.01$ and $\zeta_{2}=\zeta_{2}^{\prime}=0.02$. This combination of parameters leads to $2: 1$ internal resonance conditions. First, note that there exist two resonance peaks around $\bar{\omega}=0.65$ with very close frequencies, while the peak of the resonance branch around 1.3 consists of unstable solutions, generated by $\lambda=-1$ bifurcations at 1.203 and 1-348. As expected, these bifurcations give rise to a periodic response with a period equal two times the forcing period. To illustrate this, $n=2$ solutions arising in that frequency range are also determined and shown. In addition, there is a branch of $n=1$ unstable solutions in the range from $\bar{\omega}=0.963$ to 0.990 , originated by a Hopf bifurcation. Again, this branch is located around the middle of the primary resonance frequencies. However, there is a qualitative difference between the response of the present system and that of the oscillators analyzed in the previous section: namely, varying the last set of parameters may result in unstable ranges near the middle of the primary resonances, generated by period doubling and not by Hopf bifurcations. For other sets of parameters, several unstable intervals may appear in the same range, some generated by period doubling and the others generated by Hopf bifurcations. For example, decreasing the damping ratios of the last example to $\zeta_{1}=\zeta_{2}=\zeta_{2}^{\prime}=0.001$, apart from an order of magnitude change of the response amplitude and a slight bilateral expansion of the Hopf generated unstable solutions (which now occupy the range of $\bar{\omega}$ from 0.955 to 0.998 ), an additional unstable range of $n=1$ solutions is generated with $\lambda=-1$ between 0.828 and 0.865 .

Similar observations are made for the response of the following example, with parameters $\mu=\beta=1, \rho=0 \cdot 4, \rho^{\prime}=0.35$ and $\zeta_{1}=\zeta_{2}=\zeta_{2}^{\prime}=0.001$, leading to 3:1 internal resonance conditions. The response diagram for this system is shown in Figure 8. Again, an unstable range of $n=1$ solutions appears through $\lambda=-1$ bifurcations at $\bar{\omega}=0.685$ and 0.732 . The $n=2$ solutions existing in that frequency range are also presented in order to illustrate that

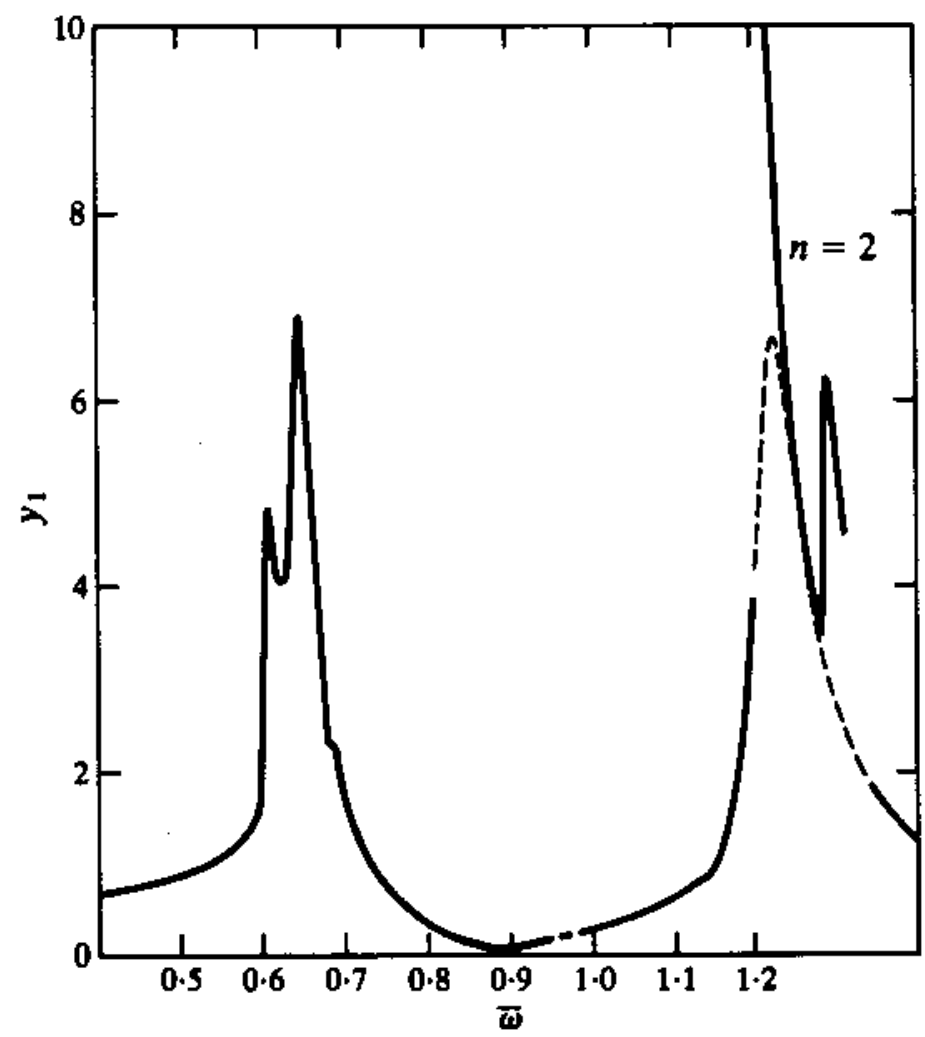

Figure 7. The response diagram of $m_{1}$ (2:1 internal resonance). 


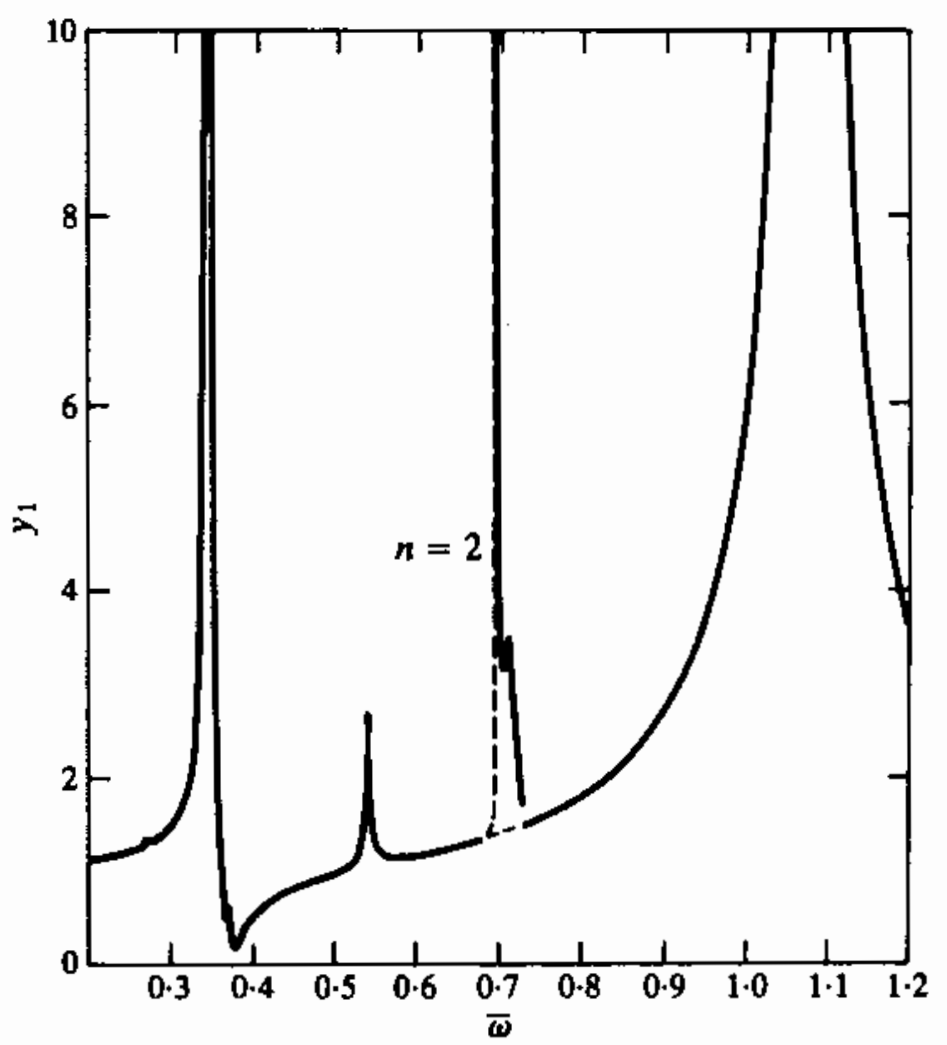

Figure 8. The response diagram of $m_{1}$ (3:1 internal resonance).

their amplitude is larger (by more than an order of magnitude near resonance) than the amplitude of the coexisting $n=1$ motions. Moreover, the $n=2$ solutions undergo saddlenode bifurcations, giving rise to a very narrow frequency range in which two stable and one unstable $n=2$ solutions coexist. Finally, note the multiple peaks for both the $n=1$ and $n=2$ branches.

As for the 2:1 case, changing the parameters may result in multiple frequency ranges involving unstable response generated by either $\lambda=-1$ or Hopf bifurcations. For

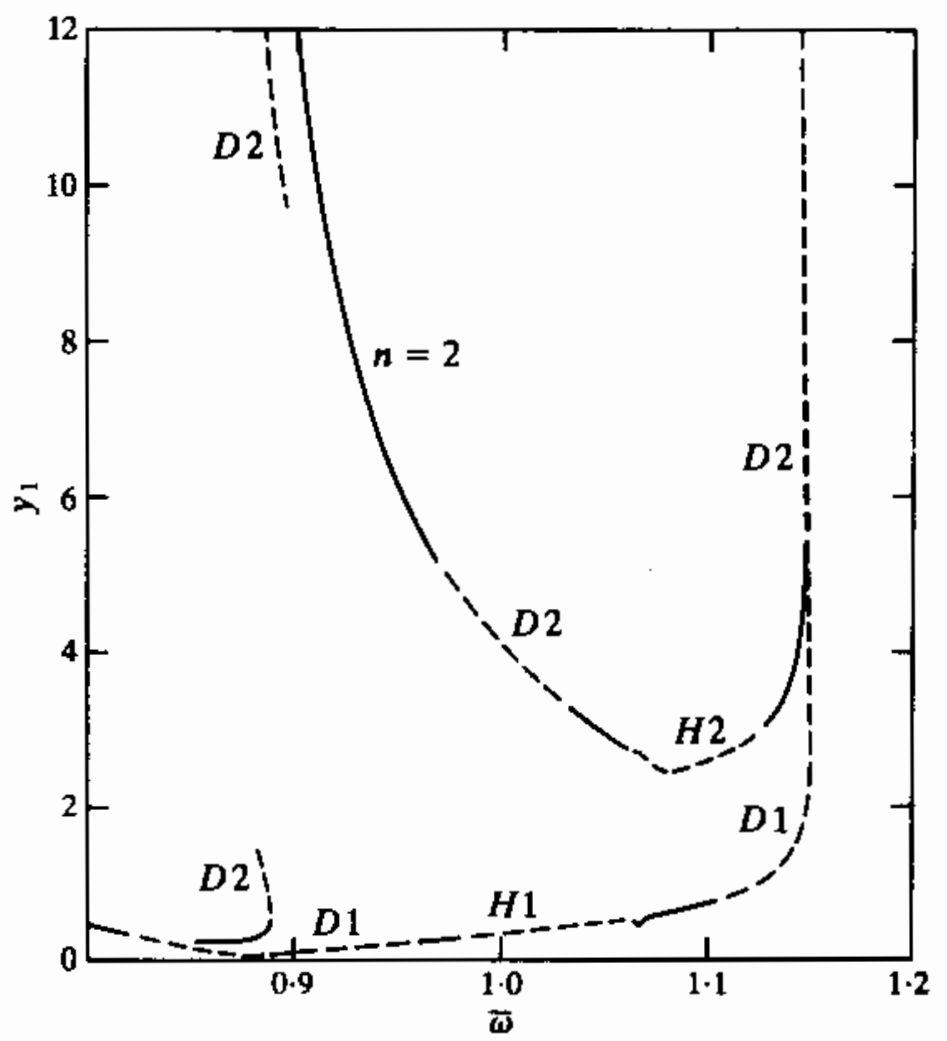

Figure 9. The response diagram of $m_{\mathrm{t}}(3: 1$ internal resonance, $\rho=0.9)$. 
example, in Figure 9 is shown the response diagram obtained for the last example between $\bar{\omega}=0.8$ and 1.2 by increasing the value of $\rho$ to 0.9 . Both $n=1$ (index 1 ) and $n=2$ (index 2) solutions are shown. The unstable branches denoted by $H$ and $D$ are generated by Hopf and period doubling bifurcations, respectively. In fact, in some cases the bifurcation takes place with a pair of complex conjugate eigenvalues equal to one, while a real eigenvalue has a value very close to -1 , indicating conditions close to codimension two bifurcations [15].

To present a small but representative sample of some of the interesting dynamics encountered in that range, in Figure 10 is shown a sequence of Poincare plots $\left(y_{2}, \dot{y}_{2}\right)$ obtained by direct integration for the parameters of the last example. For that system, a Hopf bifurcation of the $n=2$ solution occurs at $\bar{\omega}=1 \cdot 1292$. Poincare plots at $\bar{\omega}=1 \cdot 130$ and 1.129 are shown in Figure 10(a). Obviously, the $n=2$ solution, represented by the two heavy dots, is replaced by two invariant circles, following the bifurcation. Moving to lower values of $\bar{\omega}$, the solution finally locks to an $n=8$ solution, as shown in Figure 10(b) for $\bar{\omega}=1 \cdot 127$. In the same figure, the stars indicate a coexisting $n=7$ solution, resulting from different starting conditions. In fact, a third steady state solution, shown in Figure 10(c),
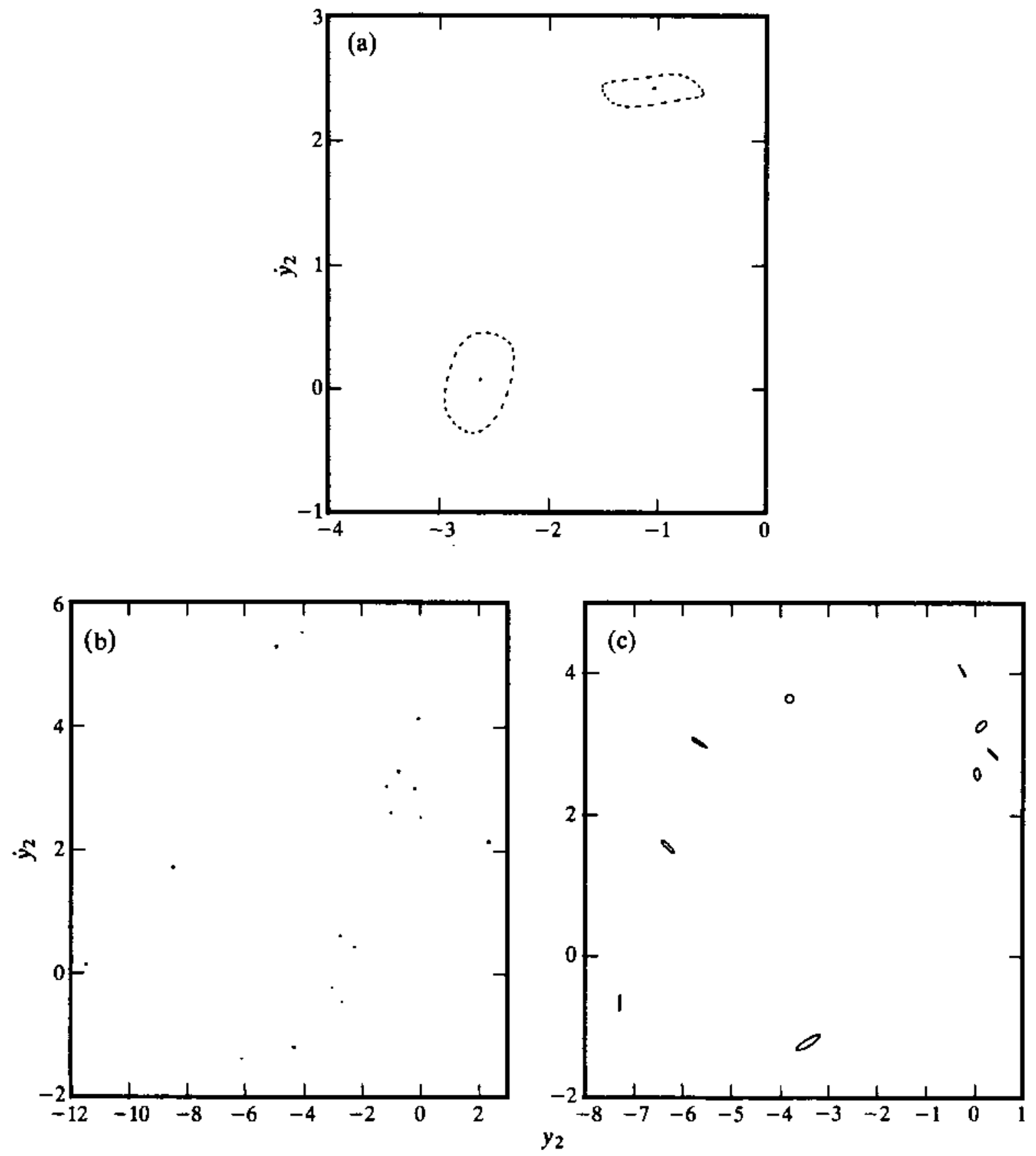

Figure 10. Poincaré sections: (a) $\bar{\omega}=1 \cdot 130$ and $1 \cdot 129$; (b) $\bar{\omega}=1 \cdot 127$; (c) $\bar{\omega}=1 \cdot 127$. 
is also determined at the same frequency. The interesting feature of this solution is that it consists of nine invariant circles, possibly originated by a Hopf bifurcation of an $n=9$ solution. When $\bar{\omega}$ is varied, each of these invariant circles undergoes changes similar to those of the single circle of Figure 5(a).

The behavior encountered in this section presents similarities and differences with the behavior of systems with asymmetric but continuous non-linearities [24]. Here, in addition to the unstable zones generated by the Hopf bifurcation between the primary resonant frequencies, there may exist zones that originated with $\lambda=-1$ bifurcations. This last type of bifurcation does not have an analog in the analysis of the steady state solutions captured in reference [24]. The reason is that the stability of those solutions is governed by linearized differential equations (flows), while here it is governed by difference equations (maps).

\section{SYNOPSIS AND CONCLUSIONS}

An investigation is carried out on the long-time response of a class of multiple-degreeof-freedom oscillators with strongly non-linear characteristics. The non-linearity of the system is modeled as a component with piecewise linear damping and stiffness properties. The solution procedure exploits the piecewise linear nature of the governing equations. Suitable solution forms are first developed for the response within the intervals where the system characteristics remain constant. Then, exact, $n$-periodic, single crossing steady state motions are determined by imposing a set of periodicity conditions. For an $N$-degree-offreedom system, this results in a system of $4 N+2$ transcendental equations. However, generalizing previous work on similar single-degree-of-freedom systems, the numerical effort is reduced to finding the solution of sets of linear equations and eventually to a single transcendental equation for the crossing time. Finally, the analytical procedure is complemented by a stability analysis which is appropriate for solutions of systems with arbitrary piecewise linear characteristics. As in the case of single-degree-of-freedom oscillators, this task is reduced to examining the propagation of errors in starting conditions within a single linear motion interval.

The present analysis provides efficient means for performing parametric studies in a large number of systems encountered in engineering practice. Here, the applicability and effectiveness of this analysis is demonstrated by examining the response of vibration absorbers with elastic stops, as well as the response of other non-linear oscillators with parameters leading to $2: 1$ and 3:1 internal resonance conditions. At the same time, the results illustrate some interesting features of the dynamics of these systems: namely, the absorber with stops is shown to possess superior technical characteristics. It provides a way in which to design non-linear absorbers and to suppress vibration levels over broader forcing frequency ranges than the conventional absorber, on both sides of the original resonant frequency. However, stability considerations in conjunction with numerical integration reveal that high amplitude beating and chaotic response may arise near the original resonance, because the coexisting periodic response may become unstable due to Hopf bifurcations. Next, similar phenomena are observed for other piecewise linear oscillators exhibiting 2:1 and 3:1 internal resonances. In these cases, zones of unstable periodic solutions are also observed in the vicinity of the middle of the primary resonances of the system. The resulting motions are again of much higher amplitude than that of the coexisting unstable $n=1$ response. Several unstable zones may exist, generated by either Hopf or period doubling bifurcations. It is shown that the Hopf bifurcation of subharmonic solutions results in multiple invariant circles. The coexistence of harmonic with large-period periodic and quasi-periodic responses is also presented. In all cases, similarities and differences in the response of systems possessing continuous non-linearities are noted. 


\section{REFERENCES}

1. S. F. MASRI 1972 Journal of Applied Mechanics 39, 563-568. Theory of the dynamic vibration neutralizer with motion limiting stops.

2. S. F. MASRI 1973 Journal of Applied Mechanics 40, 127-132. Steady-state response of a multidegree system with an impact damper.

3. L. E. Galhoud, S. F. MASRI and J. C. Anderson 1987 Journal of Applied Mechanics 54, 215225. Transfer function of a class of nonlinear multidegree of freedom oscillators.

4. C. C. Fu and B. PAUL 1969 Journal of Engineering for Industry 91, 1175-1179. Dynamic stability of a vibrating hammer.

5. M. M. Nigm and A. A. Shabana 1983 Journal of Sound and Vibration 89, 541-557. Effect of an impact damper on a multidegree of freedom system.

6. J. M. T. Thompson, A. R. BokAiAN and R. GHAFFARI 1983 IMA Journal of Applied Mathematics 31, 207-234. Subharmonic resonances and chaotic motions of a bilinear oscillator.

7. S. W. Shaw and P. J. Holmes 1983 Journal of Sound and Vibration 90, 129-155. A periodically forced piecewise linear oscillator.

8. J. Shaw and S. W. Shaw 1989 Journal of Applied Mechanics 56, 168-174. The onset of chaos in a two degree of freedom impacting system.

9. M. Kar Yeaclis and T. K. CAUghey 1989 Journal of Applied Mechanics 56, 926-940. Stability of a semi-active impact damper, parts 1 and 2.

10. Y. B. Kim and S. T. NOAH 1991 Journal of Applied Mechanics 58, 545-553. Stability and bifurcation analysis of oscillators with piecewise linear characteristics: a general approach.

11. A. Kahraman and R. Singh 1991 Journal of Sound and Vibration 144, 469-506. Non-linear dynamics of a geared rotor-bearing system with multiple clearances.

12. S. Natsiavas 1990 Journal of Sound and Vibration 141, 97-102. Stability and bifurcation analysis for oscillators with motion limiting constraints.

13. S. Natsiavas 1990 International Journal of Nonlinear Mechanics 25, 535-554. On the dynamics of oscillators with bilinear damping and stiffness.

14. S. Natsiavas and H. GonZalez 1992 Journal of Applied Mechanics 59. Vibration of harmonically excited oscillators with asymmetric constraints.

15. J. Guckenheimer and P. Holmes 1983 Nonlinear Oscillations, Dynamical Systems and Bifurcations of Vector Fields. New York: Springer-Verlag.

16. J. OR MONDROYd and J. P. Den HARTOG 1928 Transactions of ASME 50, 9-15. The theory of the dynamic vibration absorber.

17. H. J. RICE 1986 Journal of Sound and Vibration 108, 526-532. Combinational instability of the nonlinear vibration absorber.

18. J. Shaw, S. W. Shaw and A. G. Haddow 1989 International Journal of Nonlinear Mechanics 24, 281-293. On the response of the nonlinear vibration absorber.

19. S. NaTSIAVAS 1992 Journal of Sound and Vibration 156, 227-245. Steady state oscillations and stability of nonlinear dynamic vibration absorbers.

20. D. G. Aronson, M. A. Chory, G. R. Hall and R. P. MCGehee 1982 Communications in Mathematical Physics 83, 303-354. Bifurcations from an invariant circle for two parameter families of maps of the plane: a computer assisted study.

21. A. Arneodo, P. H. Coullet and E. A. Spiegel 1983 Physics Letters 94A, 1-6. Cascade of period doublings of tori.

22. K. KANEKo 1984 Progress of Theoretical Physics 72, 202-215. Oscillation and doubling of torus.

23. J. Miles 1984 Physica D 11, 309-323. Resonant motion of a spherical pendulum.

24. P. R. Sethna and A. K. BAJAJ 1978 Journal of Applied Mechanics 45, 895-902. Bifurcations in dynamical systems with internal resonance.

25. J. M. Johnson and A. K. BAJAJ 1989 Journal of Sound and Vibration 128, 87-107. Amplitude modulated and chaotic dynamics in resonant motion of strings.

26. A. K. BAJAJ and S. Tousi 1990 International Journal of Nonlinear Mechanics 25, 625-642. Torus doublings and chaotic amplitude modulations in a two degree-of-freedom resonantly forced mechanical system. 